\title{
Online commentary during the physical examination: a communication tool for avoiding inappropriate antibiotic prescribing?
}

\author{
Rita Mangione-Smith ${ }^{\mathrm{a}, *}$, Tanya Stivers ${ }^{\mathrm{a}}$, Marc Elliott ${ }^{\mathrm{b}}$, Laurie McDonald ${ }^{\mathrm{b}}$, \\ John Heritage ${ }^{\mathrm{c}}$ \\ a Department of Pediatrics, 12-365 Marion Davies Children's Center, University of California, Los Angeles, 10833 LeConte Avenue, \\ Los Angeles, CA 90095-1752, USA \\ ${ }^{\mathrm{b}}$ RAND, 1700 Main St., Santa Monica, CA 90401, USA \\ ${ }^{\mathrm{c}}$ University of California, Los Angeles, Department of Sociology, 264 Haines Hall, 375 Portola Plaza, Los Angeles, CA 90095-1551, USA
}

\begin{abstract}
A previously identified communication behavior, online commentary, is physician talk that describes what he/she is seeing, feeling, or hearing during the physical examination of the patient. The investigators who identified this communication behavior hypothesized that its use may be associated with successful physician resistance to perceived or actual patient expectations for inappropriate antibiotic medication. This paper examines the relationship between actual and perceived parental expectations for antibiotics and physician use of online commentary as well as the relationship between online commentary use and the physician's prescribing decision. We conducted a prospective observational study in two private pediatric practices. Study procedures included a pre-visit parent survey, audiotaping of study consultations, and post-visit surveys of the participating physicians. Ten pediatricians participated (participation rate $=77 \%$ ) and 306 eligible parents participated (participation rate $=86 \%$ ) who were attending sick visits for their children with upper respiratory tract infections between October 1996 and March 1997. The main outcomes measured were the proportion of consultations with online commentary and the proportion of consultations where antibiotics were prescribed. Two primary types of online commentaries were observed: (1) online commentary suggestive of a problematic finding on physical examination that might require antibiotic treatment ("problem' online commentary), e.g., "That cough sounds very chesty"; and (2) online commentary that indicated the physical examination findings were not problematic and antibiotics were probably not necessary ('no problem' online commentary), e.g., "Her throat is only slightly red". For presumed viral cases where the physician thought the parent expected to receive antibiotics, if the physician used at least some 'problem' online commentary, he/she prescribed antibiotics in $91 \%(10 / 11)$ of cases. Conversely, when the physician exclusively employed 'no problem' online commentary, antibiotics were prescribed $27 \%(4 / 15)$ of the time $(p=0.07)$. Use of 'no problem' online commentary did not add significantly to visit length. 'No problem' online commentary is a communication technique that may provide an effective and efficient method for resisting perceived expectations to prescribe antibiotics. (C) 2002 Elsevier Science Ltd. All rights reserved.
\end{abstract}

Keywords: Doctor-parent communication; Antibiotic prescribing; Online commentary

\footnotetext{
*Corresponding author. Tel.: +1-310-206-1008; fax: +1310-206-4855.

E-mail address: ritams@ucla.edu (R. Mangione-Smith).
}

\section{Background}

Upper respiratory tract infections (URTI) are a leading cause of acute morbidity, visits to physicians, and school absenteeism among children in the United 
States (Benson \& Marano, 1994). Despite a large body of evidence that antibiotics have a limited role in treating a majority of URTIs (Damoiseaux, Van Balen, Hoes, Verheij, \& De Melker, 2000; Kaiser et al., 1996), an estimated $38 \%$ of children diagnosed with the common cold leave the doctor's office with an antibiotic prescription (Nyquist, Gonzales, Steiner, \& Sande, 1998) while nearly $100 \%$ diagnosed with acute otitis media receive antibiotics (Froom et al., 1997). Approximately $\$ 40$ million is spent annually in the United States on antibiotics for the common cold (Mainous, Hueston, \& Clark, 1996). Frequent antibiotic use is a risk factor for the development of drug-resistant strains of bacteria both at the individual and community levels (Lipsitch, 2001; Deeks et al., 1999), which are costly (Holmberg, Solomon, \& Blake, 1987), more difficult to treat (Watanabe et al., 2000; Dagan, 2000), and result in increased mortality of hospitalized adults (Feikin et al., 2000).

Efforts to reduce inappropriate antibiotic prescribing have generally focused on educating physicians. However, there are many instances where the physician is aware of the problems related to appropriateness, safety, efficacy, and cost but these factors are outweighed by others such as perceived patient/parental expectations or lack of time to negotiate a different management plan (Mangione-Smith, McGlynn, Elliott, Krogstad, \& Brook, 1999; Butler, Rollnick, Pill, Maggs-Rapport, \& Stott, 1998). Intervention trials that do not take these other issues into account are likely to fail in changing prescribing behavior.

A recent observational study identified a communication technique used by physicians who often successfully avoided inappropriate antibiotic prescribing (Heritage \& Stivers, 1999). This technique, called online commentary, is talk that describes what the physician is seeing, feeling, or hearing while examining the patient. The study found that online commentary was overwhelmingly used to characterize patient signs as mild or nonexistent, and that it was strongly associated with "no problem' diagnoses, i.e., diagnoses for which antibiotic treatment would not be indicated. The study identified two basic kinds of online commentary: (1) 'no problem' online commentary, which indicated that the physician was not finding any problems on the physical examination that would be likely to require antibiotics, e.g., "Her nose is only slightly congested. It really doesn't look bad at all" or "Your ears look fine", and (2) 'problem' online commentary, which indicated the physician had found a problem that would likely need to be treated with antibiotics, e.g., "There's definitely white spots on her tonsils". The investigators concluded that 'no problem' online commentary may build a case for a 'no problem' diagnostic evaluation and/or a 'symptomatic' treatment recommendation, e.g., recommending over-the-counter medi- cations, thus pre-empting parent or patient resistance to such evaluations.

Although the study completed by Heritage and Stivers (1999) was not specifically focused on the issue of antibiotic prescribing, they observed that a substantial number of cases of online commentary addressed patient complaints where antibiotics were potentially a treatment option (Heritage \& Stivers, 1999). In a subset of 50 acute and follow-up pediatric visits where antibiotic treatment was an option, 'no problem' online commentary occurred in $76 \%$ of the cases. In three-quarters of these cases, antibiotics were not prescribed.

The objective of the current study was to gain a better understanding of how physicians use online commentary in response to actual and perceived parental expectations for antibiotics and to learn whether online commentary has potential as a communication technique that physicians could use to resist pressure for inappropriate antibiotic prescriptions. The specific aims of the study were to: (1) examine the relationship between actual and perceived parental expectations for antibiotics and physicians' use of online commentary; (2) examine the relationships between online commentary use and the physician's prescribing decision; and (3) to determine whether use of online commentary substantially increased visit length.

\section{Methods}

\section{Study design}

We collected data in two private pediatric practices. Ten pediatricians participated (77\% participation rate). All of the physicians ( 3 of 3 ) in one practice participated while in the second practice all of the full time physicians participated ( 5 of 5 ). Three of five part time physicians in the second practice did not participate. These three physicians each work one half day per week and see approximately $5 \%$ of the total patient population in the practice.

Between 10/96 and 3/97, we enrolled 306 parents (86\% participation rate) who sought care for their children's respiratory illnesses. Parents were eligible to participate in the study if they spoke and read English, their child was between 2 and $10 \mathrm{yr}$ old, was being seen for upper respiratory tract infection symptoms (cough, rhinorrhea, throat pain, ear pain, or ear tugging), had not been on antibiotics for the prior two weeks, and was seeing a participating physician. Parents who participated were older $($ mean $=38 \mathrm{yr})$, highly educated $($ mean $=16 \mathrm{yr})$, and had high incomes $(75 \%$ had annual incomes greater than $\$ 50,000$ ). Non-whites comprised one-third of the sample, and $60 \%$ were enrolled in managed care plans (Mangione-Smith et al., 1999). 
The University of California, Los Angeles Institutional Review Board, approved all study procedures involving human subjects. The study design and survey data collection methods have been described in detail elsewhere (Mangione-Smith et al., 1999). Briefly, parents completed a 15 -item pre-visit expectations inventory which included one item about whether they thought it was necessary for the physician to prescribe antibiotics. Each item on the expectations inventory was scored on a 5-point Likert scale (response set: "Definitely Necessary"(1), "Probably Necessary"(2), "Uncertain"(3), "Probably Unnecessary"(4), and "Definitely Unnecessary"(5)). Response to an item was then dichotomized so that a score of 1 or 2 was categorized as a positive expectation and a score of 3-5 was categorized as a negative expectation. Physicians completed a post-visit questionnaire to indicate diagnosis, treatment, and whether they believed the parent expected antibiotics for their child. For diagnosis and treatment, the physicians could either select one of several choices provided on a checklist or could write-in their diagnoses and prescribed treatments. The physician was also asked to respond to the statement, "This parent expected me to prescribe antibiotics". The item was scored on a 5-point Likert scale (response set: "Strongly Agree"(1), "Somewhat Agree"(2), "Uncertain"(3), "Somewhat Disagree"(4), and "Strongly Disagree"(5)). A dichotomous variable was created with a score of 1 or 2 representing a positive physician belief that the parent expected antibiotics and a score of 3-5 representing that the physician did not believe the parent expected antibiotics.

\section{Online commentary coding scheme}

With informed consent from both the participating parents and physicians, the study consultations were audiotaped. The audiotaped consultations were transcribed and coded for communication events of interest by one of the authors (TS), using a previously developed interaction analysis coding scheme (Table 1). The coder was blinded to the outcome of each encounter with regard to antibiotic prescribing until she came to the section of the transcript where treatment was discussed. Almost all online commentary preceded treatment discussions.

For each of the study consultations, we first analyzed whether or not online commentary occurred. We then coded each online comment on two additional dimensions. First, each online comment was assessed to determine whether or not it indicated the presence of an examination finding (e.g., "His throat is definitely red") or the absence of an examination finding ("I don't see any fluid in his ear"). Second, each present online comment was assessed to determine whether or not the comment indicated a problem. 'Problem' online

Table 1

Online coding scheme

\begin{tabular}{|c|c|c|}
\hline Communication behavior & Description & Examples \\
\hline \multirow[t]{4}{*}{ Occurence of online commentary } & $\begin{array}{l}\text { Comments during the physical } \\
\text { examination-prior to an official } \\
\text { diagnosis-about the presence or } \\
\text { absence of signs }\end{array}$ & "There's some pus on her tonsils" \\
\hline & & "This ear is quite red" \\
\hline & & "His chest sounds clear" \\
\hline & & "I don't feel any swollen glands" \\
\hline \multicolumn{3}{|l|}{ Problem online commentary } \\
\hline \multirow[t]{2}{*}{ (i) Present sign } & $\begin{array}{l}\text { Online comment reports a sign which } \\
\text { can be seen/heard/felt }\end{array}$ & "Her ear is draining fluid" \\
\hline & & "There's inflammation there" \\
\hline \multirow[t]{3}{*}{$\begin{array}{l}\text { (ii) Problematic evaluation of a } \\
\text { present sign }\end{array}$} & $\begin{array}{l}\text { Online comment that negatively } \\
\text { assesses an exam finding }\end{array}$ & "His cough sounds pretty chesty" \\
\hline & & "Her lungs sound awful" \\
\hline & & "That ear looks terrible" \\
\hline \multicolumn{3}{|l|}{ No problem online commentary } \\
\hline \multirow[t]{2}{*}{ (i) Absent sign } & $\begin{array}{l}\text { Online comment reports that a sign } \\
\text { cannot be seen } / \text { heard/felt }\end{array}$ & "I don't see anything in that ear" \\
\hline & & "I don't hear any crackles in her lungs" \\
\hline \multirow[t]{3}{*}{$\begin{array}{l}\text { (ii) Non-problematic evaluation of a } \\
\text { present sign }\end{array}$} & $\begin{array}{l}\text { Online comment that positively } \\
\text { assesses an exam finding }\end{array}$ & "Her nose isn't too bad" \\
\hline & & "This is only a little bit red" \\
\hline & & "Just a slight wheeze today" \\
\hline
\end{tabular}


commentary constituted the physician discussing a sign that was present and problematic. 'No problem' online commentary constituted the physician discussing either the absence of a sign on physical examination or the presence of a sign that he/she felt was not problematic. For example, the physician says, "I mean I can see a little bit of fluid, but it's really nothing to worry about". Here the physician reports on a present examination finding which is treated as not problematic, i.e., not indicative of infection or of a condition requiring antibiotic treatment. This particular statement would be coded as 'no problem' online commentary.

We evaluated each consultation regarding the types of online used by the physician, i.e., exclusively 'no problem' online, exclusively 'problem' online, or a combination of both types which we coded as at least some 'problem' online commentary. For analysis purposes, consultations with exclusive use of 'problem' online commentary and consultations with at least some 'problem' online commentary were combined since they behaved virtually identically in preliminary analyses. We also evaluated the overall frequency of online commentary across the data sample, and further explored interphysician variability of its use.

Forty-three $(15 \%)$ randomly selected records were independently coded by a second coder $(\mathrm{JH})$ who was also blinded to the consultation outcomes with regards to antibiotic prescribing. This was done to evaluate inter-rater reliability of the coding scheme for this data set. Reliability was assessed using the Kappa statistic. In the current study, the interaction analysis coding scheme had excellent inter-rater reliability, with Kappas ranging between 0.78 to 0.98 for the various codes.

\section{Analytic methods}

The coded audiotape data were merged with the survey data to examine the relationships between (1) actual and perceived parental expectations for antibiotics and online commentary use by physicians and (2) online commentary use and antibiotic prescribing patterns. Audiotapes were also directly assessed for visit length. Bivariate relationships between categorical variables were tested using simple or multiple logistic regression, correcting for clustering of encounters within physicians. Bivariate relationships between categorical and continuous variables were tested using least squares linear regression, correcting for clustering of encounters within physicians.

\section{Results}

For the current analysis, data were complete for 284 consultations (93\%). Twelve of these cases were assigned diagnoses that were neither viral nor bacterial (e.g., abdominal pain, ear wax impaction, allergic rhinitis). This group of 12 consultations was excluded from further analyses. Of the remaining 272 consultations, $54 \%(n=154)$ of the children had presumed viral illnesses where the physician assigned one of the following diagnoses: viral URI, viral pharyngitis, bronchitis, croup, and viral stomatitis. Forty-one percent $(n=118)$ had presumed bacterial illnesses where the physician assigned one of the following diagnoses: acute otitis media, otitis media with effusion, otitis externa, bacterial (streptococcal) pharyngitis, and sinusitis. Bronchitis was coded as a viral diagnosis because most cough illness of short duration (7 days or less) is thought not to be bacterial in origin (Cherry, 1998; O'Brien et al., 1998). Four of the 24 children with presumed bronchitis had been ill for 7 days or more. Recoding these cases as bacterial did not significantly alter the reported results. Although not all cases of otitis media with effusion (OME) are bacterial in origin, we chose to classify this problem as a bacterial illness because the Agency for Healthcare Research and Quality OME Clinical Practice Guideline suggests that antibiotics are an appropriate treatment option for this condition regardless of how long the child has had it (Stool, Berg, \& Berman, 1994).

A previous analysis of these data revealed that the physicians were generally not good predictors of parental expectations. Fifty percent of the parents reported that they expected to receive antibiotics. Physicians perceived that antibiotics were desired in $34 \%$ of consultations overall. They were correct about parental expectations $73 \%$ of the time when parents did not expect antibiotics and $41 \%$ of the time when parents expected antibiotics. This degree of agreement is just slightly better than chance $(P<0.05 ;$ kappa $=0.14)$ (Mangione-Smith et al., 1999).

All 10 participating physicians used online commentary during their study consultations. However, they varied significantly in how often they used it, with individual physicians employing online commentary in $60-100 \%$ of their study visits $(p<0.001$, comparing these proportions among the 10 participating physicians). Among consultations where physicians used 'no problem' online commentary exclusively, e.g., "Sara's throat is only a little red. No white spots", antibiotics were prescribed $45 \%$ of the time. In contrast, when physicians used at least some 'problem' online commentary, e.g., "His cough sounds very chesty", antibiotics were prescribed $66 \%$ of the time $(p<0.001)$.

Physicians in our sample used online commentary in more than $90 \%$ of consultations when the diagnosis was bacterial (Table 2, column 1, rows 1a and 1b). For viral cases, they used it $93 \%$ of the time when they believed the parent expected antibiotics (Table 2, column 1, and row 2a). However, in viral cases where physicians did not believe that antibiotics were desired, they 
Table 2

Use of online commentary, by diagnosis assigned and physician perceptions of parental expectations for antibiotics ${ }^{\mathrm{a}}$

Type of online commentary used

Type of diagnosis assigned

(1) Proportion of $\quad$ (2) Proportion of consultations where online consultations where was used exclusively 'no problem' online was used
(3) Proportion of consultations where at least some 'problem' online was used

(1) Bacterial $(n=118)$

(a) Physician thinks parent

expects antibiotics $(n=61)$

(b) Physician does not think $93 \%(57 / 61)$

$42 \%(26 / 61)$

$51 \%(31 / 61)$

parent expects antibiotics

$95 \%(54 / 57)$

$46 \%(26 / 57)$

$49 \%(28 / 57)$

$(n=57)$

(2) Viral (154)

(a) Physician thinks parent

expects antibiotics $(n=28)$

(b) Physician does not think

parent expects antibiotics

$93 \%(26 / 28)$

$54 \%(15 / 28)$

$39 \%(11 / 28)$

$76 \%(96 / 126)$

$51 \%(64 / 126)$

$25 \%(32 / 126)$

$(n=126)$

\footnotetext{
${ }^{\mathrm{a}}$ Note: Comparisons in Table 2 reflect multiple logistic regression, with corrections for clustering of encounters within physician. Column 1: Rows 1a, $1 \mathrm{~b}$, and $2 \mathrm{a}$ do not significantly differ $(p>0.30$ for all pairs). Row $2 \mathrm{~b}$ significantly differs from rows $1 \mathrm{a}$ and $1 \mathrm{~b}$ $(p<0.05$ in both case), and differs with marginal significance from row $2 \mathrm{a}(0.05<p<0.10)$.

Column 2: No pairs of rows significantly differ ( $p>0.30$ for all pairs).

Column 3: Rows $1 \mathrm{a}, 1 \mathrm{~b}$, and $2 \mathrm{a}$ do not significantly differ ( $p>0.30$ for all pairs). Row $2 \mathrm{~b}$ significantly differs from rows $1 \mathrm{a}$ and $1 \mathrm{~b}$ $(p<0.05$ in both cases), but does not significantly differ from row $2 \mathrm{a}(p=0.18)$ all tests two-sided.
}

significantly decreased their use of online commentary $(p<0.05$, Table 2, column $1,76 \%$ of cases versus $93-95 \%$ of cases).

Exclusive use of 'no problem' online commentary was no more likely to occur during consultations where a viral diagnosis was assigned than it was for consultations where a bacterial diagnosis was assigned ( $p>0.30$, Table 2, column 2). When physicians perceived that parents expected antibiotics within each diagnostic category, they were also no more likely to exclusively use 'no problem' online commentary than they were in the absence of these perceptions $(p>0.30$, Table 2 , column 2).

Use of at least some 'problem' online commentary was similar in frequency for all bacterial cases and for viral cases where physicians thought parents expected antibiotics ( $p>0.30$, Table 2 , column 3, rows $1 \mathrm{a}, 1 \mathrm{~b}$, and $2 a)$. In contrast, when viral diagnoses were assigned and physicians did not think that parents expected antibiotics, their use of 'problem' online commentary decreased significantly compared to bacterial consultations $(p<0.05$, Table 2, column $3,25 \%$ of cases compared to $49-51 \%$ of cases ).

Unlike physician perceived expectations, actual parent expectations were not associated with the physician's use of either 'problem' or 'no problem' online commentary ( $p>0.30$, data not shown).

Prescribing antibiotics during viral illness consultations was associated with the type of online commentary used. Among consultations where viral diagnoses were made, physicians thought parents expected to receive antibiotics $18 \%$ of the time ( 28 of 154 consultations). For two of these cases, no online commentary was used and no antibiotics were prescribed. For the remaining 26 consultations, when physicians exclusively used "no problem' online commentary $(n=15)$ (e.g., "Her ears look fine") they prescribed antibiotics $27 \%$ of the time $(4 / 15)$. In contrast, when physicians used at least some 'problem' online commentary $(n=11)$ (e.g., "His nasal discharge is really thick and green") they prescribed in $91 \%(10 / 11)$ of cases $(p=0.07$, Table 3$)$.

The median visit length in this study was $4 \mathrm{~min}$. Encounters where antibiotics were prescribed were $31 \mathrm{~s}$ shorter on average than encounters where no prescription was provided ( $p=0.11$ ). Use of 'no problem' online commentary did not significantly increase visit length.

\section{Discussion}

\section{Determinants of antibiotic prescribing}

The decision to prescribe antibiotics is complex and involves multiple factors. Among these are the patient's age (Schwartz, Freij, Ziai, \& Sheridan, 1997), the duration and worsening of symptoms (Davy, Dick, \& Munk, 1998), physical examination findings (Dosh, Hickner, Mainous, \& Ebell, 2000; Le Saux, Pham, 
Table 3

Proportion of viral cases where physicians prescribed antibiotics when they thought that parents expected them ${ }^{\mathrm{a}}$

\begin{tabular}{ll}
\hline Type of online commentary used & $\begin{array}{l}\text { Proportion of viral cases where } \\
\text { antibiotics were prescribed }\end{array}$ \\
\hline Exclusively "no problem" & $27 \%(4 / 15)$ \\
At least some "problem" & $91 \%(10 / 11)$ \\
\hline${ }^{a}$ Note: The comparison in Table 3 reflects multiple logistic regression, with corrections for clustering of encounters within physician. \\
$P=0.067$.
\end{tabular}

Bjornson, \& Pitters, 1999), perceived parental expectations for antibiotics (Watson et al., 1999; MangioneSmith et al., 1999), concerns related to maintaining a positive doctor-patient/parent relationship (Butler et al., 1998), the parent's need to return to work (lack of "sick daycare" available for their child) (Barden, Dowell, Schwartz, \& Lackey, 1998), concern about adverse outcomes if treatment is withheld (Dosh et al., 2000; Butler et al., 1998), physician demographics, and physician specialty (Mainous, Hueston, \& Love, 1998; Schwartz et al., 1997).

In a previous analysis of these data, we found that physicians prescribed antibiotics for presumed viral illnesses $62 \%$ of the time when they thought parents expected antibiotics versus $7 \%$ of the time when they did not think that antibiotics were desired (Mangione-Smith et al., 1999). In a survey conducted by the Centers for Disease Control and Prevention, 58\% of the physicians similarly reported their decisions to prescribe antibiotics for children with upper respiratory infections were influenced by parental pressure (Watson et al., 1999). Butler et al. reported that the prescribing decision is strongly influenced by physician concerns related to preserving and building the doctor-patient/parent relationship. Clinicians generally prefer to meet patients' expectations when reasonable to do so even if their decision goes against published effectiveness research (Butler et al., 1998). This line of research indicates that interventions to decrease antibiotic over-prescribing for URTI, must address both psychosocial factors and the doctor-parent interaction as key elements influencing the decision to prescribe. For an intervention to be effective it will need to go beyond traditional educational campaigns and address the content of the doctorparent/patient interaction itself.

\section{Perceived expectations, doctor-parent communication, and the decision to prescribe}

Most textbooks of clinical practice make reference to talk during the physical examination. Billings and Stoeckle (1989) recommend that, "when the examination is normal, let the patient know. Everyone appreciates this good news, both during the examination and at the end of the encounter", (Billings \& Stoeckle, 1989). They suggest that talking during the examination reduces patient mystification and communicates respect by involving the patient in the care process. Zoppi suggests that talk during the examination can reassure the patient: "physical findings should be described to the patient, who otherwise may misinterpret a squint and silence as cues that something ... is horribly wrong (Zoppi, 1997). Such feedback is probably better than attempting to distract the patient with social small talk, especially if the patient is anxious".

No previous studies have attempted to associate this type of communication behavior with physicians' perceptions of parent expectations for antibiotics or with their prescribing decisions. In this study, when physicians exclusively used 'no problem' online commentary to describe their physical examination findings to parents, they were significantly less likely to prescribe antibiotics ( $45 \%$ of visits) than if they used 'problem' online commentary $(66 \%$ of visits; $p<0.001)$. This finding is consistent with previous research that suggested a possible link between use of 'no problem' online commentary and less antibiotic prescribing (Heritage \& Stivers, 1999).

Although physicians varied substantially in their frequency of online commentary use, only the use of 'problem' online commentary was associated with their perceptions of parent expectations for antibiotics. In viral cases where physicians did not think the parent expected antibiotics, they were significantly less likely to use 'problem' online commentary suggesting a condition that was potentially treatable with antibiotics (e.g., "He has a chesty cough") (Table 2, column 3, row 2b). Thus, in consultations for viral illness when they did not think the parent expected antibiotics, the study physicians decreased the frequency with which they described problematic medical signs that could raise expectations for antibiotic treatment.

Physician perceptions of parent expectations for antibiotics were not associated with differential use of 'no problem' online commentary. In consultations where physicians exclusively used 'no problem' online commentary, they used it with equal frequency whether the diagnosis was bacterial or viral and whether they perceived expectations to prescribe antibiotics or not. So although 'no problem' online commentary shows promise for avoiding inappropriate prescribing (Table 3), physicians in our study were not applying it 
more intensively during consultations where it might have been the most useful, i.e., viral cases where they perceived parents as expecting antibiotics.

In a study that did not take perceived parental expectations into account, Nyquist et al. found that physicians in the United States prescribed antibiotics for $38 \%$ of children with the common cold (Nyquist et al., 1998). In the current study, we examined a subset of viral upper respiratory tract infection cases where physicians perceived a parental expectation for antibiotics. We found that inappropriate prescribing increased markedly when perceived expectations were taken into consideration. In our study, for viral cases where physicians thought that parents expected antibiotics $(n=28)$, they prescribed $50 \%$ of the time. However, if they exclusively used 'no problem' online commentary to describe their physical examination findings $(n=15)$, they were much less likely to prescribe ( $27 \%$ of cases, Table 3$)$. In contrast, if they used any 'problem' online commentary in these cases $(n=11)$, they were much more likely to prescribe ( $91 \%$ of cases). This finding supports the suggestion that 'no problem' online commentary builds a prospective case against a 'problem' diagnosis and facilitates the subsequent denial of antibiotics. Thus it appears that if physicians could more consciously and consistently apply 'no problem' online commentary in those situations where it is most likely to be effective, much inappropriate antibiotic prescribing might be avoided.

For an intervention to be successful in making longterm changes in prescribing patterns, it needs to be feasible and practical to implement. Evaluations of previous interventions developed to improve prescribing decisions reveal that most of these strategies are only minimally effective in the long term (Davis, Thompson, Oxman, \& Haynes, 1995) or are too costly and impractical to implement on a large scale (Avorn \& Soumerai, 1983). An intervention that trains physicians to augment and more consistently use a communication behavior that many of them may already be employing at least occasionally would be feasible to implement from both a cost and logistical standpoint and should have a long-lasting effect when applied to physicians in training. In this study, using 'no problem' online commentary is a "time-neutral" communication technique. Thus it could be both an effective and a timeefficient technique physicians could use to resist pressure to prescribe antibiotics inappropriately.

\section{Limitations}

Because our study was done in one geographic location with a small and relatively homogeneous group of parents and physicians, we do not know whether our findings would generalize to other settings with parents from different backgrounds. Additionally, because this is an observational study we cannot draw any conclusions about causation. We may have introduced measurement error by depending on physician and parent self-reports for some of the independent variables under consideration.

\section{Conclusions}

Inappropriate antibiotic prescribing is a critical issue worldwide. The resulting escalation in bacterial resistance is responsible for increased morbidity, mortality, and health care costs. 'No problem' online commentary may be an effective and efficient way for physicians to resist perceived pressure to prescribe antibiotics. Further research is needed to determine whether training physicians to consciously increase their use of this communication technique can decrease rates of inappropriate antibiotic prescribing.

\section{Acknowledgements}

Project funding: Agency for Healthcare Research and Quality; Grant \#: RO1 HS10187-01

\section{References}

Avorn, J., \& Soumerai, S. B. (1983). Improving drug-therapy decisions through educational outreach: A randomized controlled trial of academically based 'detailing'. New England Journal of Medicine, 308, 1457-1463.

Barden, L. S., Dowell, S. F., Schwartz, B., \& Lackey, C. (1998). Current attitudes regarding use of antimicrobial agents: Results from physicians' and parents' focus group discussions. Clinical Pediatrics, 37, 665-672.

Benson, V., \& Marano, M.A. (1994). Current estimates from the National Health Interview Survey. Vital Health Statistics Series 10 (189).

Billings, J. A., \& Stoeckle, J. D. (1989). The clinical encounter: A guide to the medical interview and case presentation. Chicago, IL: Year Book Medical Publishers.

Butler, C. C., Rollnick, S., Pill, R., Maggs-Rapport, F., \& Stott, N. (1998). Understanding the culture of prescribing: Qualitative study of general practitioners' and patients' perceptions of antibiotics for sore throats. British Medical Journal, 317, 637-642.

Cherry, J. D. (1998). Lower respiratory tract infections, acute bronchitis. In R. D. Feigin, \& J. D. Cherry (Eds.), Textbook of pediatric infectious diseases (pp. 242-245). Philadelphia, PA: W.B. Saunders.

Dagan, R. (2000). Clinical significance of resistant organisms in otitis media. Pediatric Infectious Disease Journal, 19, 378-382.

Damoiseaux, R., Van Balen, F., Hoes, A., Verheij, T., \& De Melker, R. A. (2000). Primary care based randomised, double blind trial of amoxicillin versus placebo for acute 
otitis media in children aged under 2 years. British Medical Journal, 320, 350-354.

Davis, D. A, Thompson, M. A., Oxman, A. D., \& Haynes, B. (1995). Changing physician performance a systematic review of the effect of continuing medical education strategies. JAMA, 274, 700-705.

Davy, T., Dick, P. T., \& Munk, P. (1998). Self-reported prescribing of antibiotics for children with undifferentiated acute respiratory tract infections with cough. Pediatric Infectious Disease Journal, 17, 457-462.

Deeks, S. L, Palacio, R., Ruvinsky, R., Kertesz, D. A., Hortal, M., Rossi, A., Spika, J. S., \& Di Fabio, J. L. (1999). Risk factors and course of illness among children with invasive penicillin-resistant Streptococcus pneumoniae. The Streptococcus pneumoniae Working Group. Pediatrics, 103, 409-413.

Dosh, S. A, Hickner, J. M., Mainous, A. G., \& Ebell, M. H. (2000). Predictors of antibiotic prescribing for nonspecific upper respiratory infections, acute bronchitis, and acute sinusitis. The Journal of Family Practice, 49, 407-414.

Feikin, D. R., Schuchat, A., Kolczak, M., Barrett, N. L., Harrison, L. H., Lefkowitz, L., McGeer, A., Farley, M. M., Vugia, D. J., Lexau, C., Stefonek, K. R., Patterson, J. E., \& Jorgensen, J. H. (2000). Mortality from invasive pneumococcal pneumonia in the era of antibiotic resistance, 1995-1997. American Journal of Public Health, 90, 223-229.

Froom, J., Culpepper, L. J. M., DeMelker, R. A., Green, L. A., Van Buchem, L., Grob, P., \& Heeren, T. (1997). Antimicrobials for acute otitis media? A review from the international primary care network. British Medical Journal, $315,98-102$.

Heritage, J., \& Stivers, T. (1999). Online commentary in acute medical visits: A method of shaping patient expectations. Social Science and Medicine, 49, 1501-1517.

Holmberg, S. D., Solomon, S. L., \& Blake, P. A. (1987). Health and economic impacts of antimicrobial resistance. Reviews of Infectious Diseases, 9, 1065-1078.

Kaiser, L., Lew, D., Hirschel, B., Auckenthaler, R., Morabia, A., Heald, A., Benedict, P., Terrier, F., Wuderli, W., Matter, L., Germann, D., Voegeli, J., \& Stadler, H. (1996). Effects of antibiotic treatment in the subset of common-cold patients who have bacteria in nasopharyngeal secretions. The Lancet, 347, 1507-1510.

Le Saux, N., Pham, B., Bjornson, C., \& Pitters, C. (1999). Antimicrobial use in febrile children diagnosed with respiratory tract illness in an emergency department. Pediatric Infectious Disease Journal, 18, 1078-1080.
Lipsitch, M. (2001). Measuring and interpreting associations between antibiotic use and penicillin resistance in Streptococcus pneumoniae. Clinical Infectious Diseases, 32, 1044-1054.

Mainous, A. G., Hueston, W. J., \& Clark, J. R. (1996). Antibiotics and upper respiratory infection: Do some folks think there is a cure for the common cold? The Journal of Family Practice, 42, 357-361.

Mainous, A. G., Hueston, W. J., \& Love, M. M. (1998). Antibiotics for colds in children. Who are the high prescribers? Archives of Pediatrics and Adolescent Medicine, 152, 349-352.

Mangione-Smith, R., McGlynn, E. A., Elliott, M. N., Krogstad, P., \& Brook, R. H. (1999). The relationship between perceived parental expectations and pediatrician antimicrobial prescribing behavior. Pediatrics, 103, 711-718.

Nyquist, A., Gonzales, R., Steiner, J. F., \& Sande, M. A. (1998). Antibiotics for children with upper respiratory infections. Journal of the American Medical Association, 280, 1401-1401.

O'Brien, K. L., Dowell, S. F., Schwartz, B., Marcy, M., Phillips, W. R., \& Gerber, M. A. (1998). Cough illness/ bronchitis-principles of judicious use of antimicrobial agents. Pediatrics, 101, S178-S180.

Schwartz, R. H., Freij, B. J., Ziai, M., \& Sheridan, M. J. (1997). Antimicrobial prescribing for acute purulent rhinitis in children: A survey of pediatricians and family practitioners. Pediatric Infectious Disease Journal, 16, 185-190.

Stool, S. E., Berg, A. O., \& Berman, S. (1994). Otitis media with effusion in young children. No. 12 of Clinical Practice Guideline. DHHS publication No.(AHCPR)94-0622.

Watanabe, H., Sato, S., Kawakami, K., Watanabe, K., Oishi, K., Rikitomi, N., Ii, T., Ikeda, H., Sato, A., \& Nagatake, T. (2000). A comparative clinical study of pneumonia by penicillin-resistant and sensitive Streptococcus pneumoniae in a community hospital. Respirology, 5, 59-64.

Watson, R. L., Dowell, S. F., Jayaraman, M., Keyserling, H., Kolczak, M., \& Schwartz, B. (1999). Antimicrobial use for pediatric upper respiratory infections: Reported practice, actual practice, and parent beliefs. Pediatrics, 104, 1251-1257.

Zoppi, K. A. (1997). Interviewing as clinical conversation. In M. B. Mengel, \& S. A. Fields (Eds.), Introduction to clinical skills: A patient-centered textbook (pp. 41-55). New York, NY: Plenum Medical Book Company. 